\title{
ON A PROBLEM IN CONFORMAL MAPPING OF SCHLICHT FUNCTIONS
}

\section{ALBERT SCHILD}

1. Introduction. It was shown by E. Study $[1], 2$ that if $w=f(z)$ $=z+\sum_{n=2}^{\infty} a_{n} z^{n}$ is regular and schlicht in the unit circle, then there exists a positive number $r_{0}$, such that the circle $|z| \leqq r_{0}$ will always be mapped into a convex region in the w-plane. Subsequently, Gronwall [2] and Nevanlinna [3] have shown that $r_{0} \geqq 2-3^{1 / 2}$ for all functions $f(z)=z+\sum_{n=2}^{\infty} a_{n} z^{n}$ regular and schlicht in the unit circle. This lower limit of $r_{0}$ is actually attained by the function $f(z)=z(1-z)^{-2}=\sum_{n=1}^{\infty} n z^{n}$. In this paper the following question will be considered: Suppose $w=f(z)=z+\sum_{n=2}^{\infty} a_{n} z^{n}$, regular and schlicht in the unit circle, maps $|z| \leqq 1$ into a domain $D$ in the w-plane which is star-like with respect to $w=0$, i.e. the domain $D$ is such that any interior point of it can be joined to the origin $w=0$ by a straight line segment which lies entirely in the domain $D$. Let $d^{*}$ be the distance of the closest point of the boundary of $D$ from $w=0$. Let $r_{0}$ be the bound of convexity (Rundungs-Schranke) of $w=f(z)$, i.e. $|z|=r_{0}$ is mapped into a convex curve by $w=f(z)$, but $|z|=r$, $r>r_{0}$, is not mapped into a convex curve any more. Let $d_{0}$ be the shortest distance from $w=0$ to $w=f\left(r_{0} e^{i \theta}\right)$. It has been conjectured that $d_{0} / d^{*} \geqq 2 / 3$ for all such functions. This lower bound of $2 / 3$ cannot be improved, since it is actually attained by the function $w$ $=f(z)=z(1-z)^{-2}$.

In this paper the truth of this conjecture will be demonstrated for certain classes of functions, while for certain other classes lower bounds for $d_{0} / d^{*}$ are obtained. The methods employed are of an elementary nature.

\section{Upper bounds for $d^{*}$ and $d_{0}$.}

LEMMA 2.1. $d^{*}<1$.

Proof. By applying Schwarz's Lemma to the inverse of $w=f(z)$ it follows immediately that $d^{*} \leqq 1$ where equality can be attained only when $w=f(z)=z$.

In a similar manner we have

Presented to the Society, April 28, 1952; received by the editors March 31, 1952.

1 Presented to the Graduate School of the University of Pennsylvania in partial fulfillment of the requirements for the degree of Doctor of Philosophy.

2 Numbers in brackets refer to the bibliography at the end of the paper. 
LEMMA 2.2. $d_{0}<r_{0}$.

3. Some lower bounds for $d_{0} / d^{*}$. It was shown by Koebe, Bieberbach, and others, that if $w=f(z)=z+\sum_{n=2}^{\infty} a_{n} z^{n}$ regular and schlicht maps the unit circle into a domain $D$, then $d^{*} \geqq 1 / 4$. It follows from the Distortion Theorem (Verzerrungs Satz) and Study's criterion for convex maps that $d_{0} \geqq 1 / 6$. These lower bounds cannot be improved for star-like maps, since they are attained by the function $f(z)$ $=z(1-z)^{-2}$ which maps the unit circle into a star-like region. We have therefore:

$$
\begin{aligned}
& 1 / 4 \leqq d^{*}<1 \\
& 1 / 6 \leqq d_{0} \leqq d^{*}
\end{aligned}
$$

and therefore:

$$
d_{0} / d^{*}>1 / 6=.166 \cdots
$$

LEMMA 3.4. $d_{0} / d^{*}>r_{0}$.

Proof. Suppose the regular and schlicht function $f(z)=z+\sum_{n-2}^{\infty} a_{n} z^{n}$ maps the unit circle onto a star-like region $D$. Consider $z=z(w)$ the inverse of $w=f(z)$. It fulfills the conditions of Schwarz's Lemma, being regular for $|w|<d^{*}$ and mapping $|w|<d^{*}$ into a region completely inside $|z|<1$. Hence

$$
|z / w|<\frac{1}{d^{*}} \quad \text { or }|w|>|z| \cdot d^{*}
$$

This relation will certainly hold for that special $z$ which maps into the closest point of the "last" convex curve, and therefore:

$$
d_{0}>r_{0} d^{*}
$$

$$
\text { or } d_{0} / d^{*}>r_{0} \text {. }
$$

COROLLARY 3.7. For the whole class of functions under consideration we have $d_{0} / d^{*}>2-3^{1 / 2}=.267 \cdots$.

COROLlary 3.8. For the class of functions for which $r_{0} \geqq 2 / 3$ we have $d_{0} / d^{*}>2 / 3$. This class of functions is not empty since e.g. $f(z)$ $=2\left\{1-(1-z)^{3 / 2}\right\} / 3$ for which $r_{0}=2 / 3$ belongs to this class.

4. Lower bounds of $d_{0} / d^{*}$ for functions of the type $f(z)=z$ $+\sum_{n=1}^{\infty} a_{n p+1} z^{n p+1}, p=2,3, \cdots$, which map the unit circle into a star-like region with $p$-fold rotational symmetry, i.e. the map is identical in each sector $k \cdot 2 \pi / p<\theta \leqq(k+1) 2 \pi / p, k=0,1,2, \cdots$, $p-1$. Consider first the special subclass

$$
f(z)=z\left(1+z^{p}\right)^{-2 / p}
$$


which map the unit circle into the complete $w$-plane slit radially from the points $w_{i}=(1 / 4)^{1 / p} e^{2 \pi i / p}, i=0,1,2, \cdots, p-1$, to infinity. For these functions we find:

4.4

$$
\begin{aligned}
d_{p}^{*} & =\left|w_{i}\right|=(1 / 4)^{1 / p} \\
r_{0, p} & =\left[p+1-\left(p^{2}+2 p\right)^{1 / 2}\right]^{1 / p} \\
d_{0, p} & =[2(p+2)]^{-1 / p}
\end{aligned}
$$

and therefore

$$
d_{0, p} / d_{p}^{*}=\left[\frac{2}{p+2}\right]^{1 / p}>\frac{2}{3} \quad \text { for } p=2,3, \cdots .
$$

Note that for $p=1$ we have $f(z)=z(1+z)^{-2}$ for which $d_{0} / d^{*}=2 / 3$. To discuss the general class of functions of the type $f(z)=z$ $+\sum_{n-2}^{\infty} a_{n p+1} z^{n p+1}$ we note the following:

LEMMA 4.6. If $w=f(z)$ is regular, schlicht and normalized in the unit circle, then so is $F(z)=\left\{f\left(z^{p}\right)\right\}^{1 / p}$.

Lemma 4.7. If $w=f(z)$ maps the unit circle onto a star-like domain with respect to $w=0$ then so does $w=F(z)=\left\{f\left(z^{p}\right)\right\}^{1 / p}$.

LEMMA 4.8. There is a 1-1 correspondence between functions $w=f(z)$ which map the unit circle into a schlicht, star-like region and functions which map the unit circle into a schlicht, star-like region with $p$-fold rotational symmetry. The correspondence can be accomplished by the relation:

$$
f(z) \leftrightarrow\left\{f\left(z^{p}\right)\right\}^{1 / p} .
$$

The proofs 4.6, 4.7, 4.8 can be found in (5). It follows then, that for these functions we have the following distortion theorem:

$$
|z|\left(1+|z|^{p}\right)^{-2 / p} \leqq\left|f\left(z^{p}\right)\right|^{1 / p} \leqq|z|\left(1-|z|^{p}\right)^{-2 / p} .
$$

These inequalities are exact since they are attained by the functions

$$
F_{p}(z)=z\left(1 \pm z^{p}\right)^{-2 / p} \text {. }
$$

By 4.3 we have $r_{0, p} \geqq\left[p+1-\left(p^{2}+2 p\right)^{1 / 2}\right]^{1 / p}$ and by direct calculation we find

$$
r_{0,7} \geqq .674 \cdots>2 / 3
$$

and since $r_{0, p}$ is an increasing function of $p$, we have:

THEOREM 4.13. If $w=f_{p}(z)$ maps the unit circle into a star-like region with $p$-fold rotational symmetry, then 
We also find

$$
d_{0,2} / d_{2}^{*}>.415 \cdots \text {, }
$$

$$
d_{0,3} / d_{3}^{*}>.501 \cdots \text {, }
$$

5. Functions of the type $f(z)=z+\sum_{n=2}^{\infty} a_{n} z^{n}, a_{n}$ real for $n$ $=2,3,4, \cdots$. Let $f(z)=z+\sum_{n=2}^{\infty} a_{n} z^{n}, a_{n}$ real, map $|z|<1$ into a star-shaped region. Let $f(z)$ fulfill, besides, the following two conditions:

$5.1\left|f\left(e^{i \theta}\right)\right|$ is nondecreasing for $0 \leqq \theta \leqq \pi$.

5.2 Let $\epsilon$ be an arbitrarily small positive quantity. Let the arc $0 \leqq \theta \leqq \theta_{0}<\pi, r=1-\epsilon$ be mapped by $w=f(z)$ into a curve of negative curvature, and the arc $\theta_{0}<\theta \leqq \pi, r=1-\epsilon$ into a curve of positive curvature. For functions of this type we have:

LEMma 5.3. For any $r, 0<r<1,\left|f\left(r e^{i \theta}\right)\right|$ is nondecreasing for $0 \leqq \theta$ $\leqq \pi$, and in particular $\min _{|z|-r}|f(z)|=f(r)$.

Proof. By 5.1 we have for $|z|=1,0 \leqq \theta \leqq \pi: \partial \ln |f(z)| / \partial \theta \geqq 0$ or $5.40 \leqq \operatorname{Re}\left\{\frac{\partial}{\partial \theta} \ln f(z)\right\}=\operatorname{Re}\left\{i z \frac{d}{d z} \ln f(z)\right\}=-\operatorname{Im}\left\{\frac{z f^{\prime}(z)}{f(z)}\right\}$.

Since $f(z)$ and $f^{\prime}(z)$ are real for real $z$, therefore 5.4 is also true on the real axis. Applying the principle of the maximum to the harmonic function $\operatorname{Im}\left\{z f^{\prime}(z) / f(z)\right\}$, we see that 5.4 is true for upper half of unit circle. Again, on account of symmetry-realness of coefficientsit is true inside the unit circle.

LEMMA 5.5. Let $|z|=r_{0}$ be the bound of convexity of $w=f(z)$. Then the first point of nonconvexity of the map of $|z|=r_{0}$ will be on the positive real axis.

Proof. Using a remark by E. Study [1] we know that the map of $|z|=r>r_{0}, r<1$, can be decomposed into a finite number of parts with positive and negative curvature respectively, and that the number of these parts cannot decrease with increasing $r$. Since, by as- 
sumption, the map of $|z|=1-\epsilon$ has only one piece of negative curvature, the map of $|z|=r<1-\epsilon$ can have at most one piece of negative curvature, and since the map of $|z|<1$ is symmetrical with respect to the real axis, the first point of nonconvexity must occur on the real axis. It cannot occur on the negative axis of reals on account of 5.2. At the same time, it follows that the points $r_{0}<z \leqq 1-\epsilon$ are also points of nonconvexity.

6. On the behavior of $f^{\prime}(z)$ for real positive $z$, for functions of the the type considered in $\$ 5$.

LEMMA 6.1. $\operatorname{Re}\left\{z f^{\prime}(z) / f(z)\right\}$ is strictly decreasing for real $z, 0<z<z_{0}$.

Proof. For the range of $z$ under consideration we have by our assumptions:

$$
\operatorname{Re}\left\{\frac{z f^{\prime}(z)}{f(z)}\right\}=\frac{z f^{\prime}(z)}{f(z)}
$$

Consider now

$$
\frac{d}{d z}\left\{\frac{z f^{\prime}(z)}{f(z)}\right\}=\frac{f^{\prime}(z)}{f(z)}\left[\frac{z f^{\prime \prime}(z)}{f^{\prime}(z)}+1-\frac{z f^{\prime}(z)}{f(z)}\right] .
$$

By [1] we have for the radius of curvature of the map of the circle $|z|=r$ by the function $w=f(z)$

$$
\frac{1}{\rho}=\frac{1}{|z|\left|f^{\prime}(z)\right|}\left[\operatorname{Re}\left\{\frac{z f^{\prime \prime}(z)}{f^{\prime}(z)}+1\right\}\right]
$$

and therefore in our case

$$
\frac{1}{\rho}=\frac{1}{z f^{\prime}(z)}\left[\frac{z f^{\prime \prime}(z)}{f^{\prime}(z)}+1\right]
$$

or

$$
\frac{z f^{\prime \prime}(z)}{f^{\prime}(z)}+1=\frac{z f^{\prime}(z)}{\rho} .
$$

Substituting this expression into 6.3, we get

$$
\frac{d}{d z}\left[\frac{z f^{\prime}(z)}{f(z)}\right]=\frac{z f^{\prime 2}(z)}{\rho f^{2}(z)}[f(z)-\rho] .
$$

We shall now show that the right-hand side of 6.7 is always $\leqq 0$. Clearly $z f^{\prime 2}(z) / \rho f^{2}(z)>0$. That $[f(z)-\rho] \leqq 0$ can best be seen from geometrical considerations. For, suppose, for $z=z^{\prime}, z^{\prime}$ real, $0<z^{\prime}<z_{0}$, 
$f(z)<\rho$. That would imply that part of the map of $|z|=z^{\prime}$ by $w=f(z)$ would lie inside the circle $|w|=f\left(z^{\prime}\right)$. But this is impossible by 5.3 . Hence, since $d\left[z f^{\prime}(z) / f(z)\right] / d z \leqq 0$ for real $z, 0<z<z_{0}, z f^{\prime}(z) / f(z)$ is decreasing for this range.

LEMMA 6.8. $z f^{\prime}(z) / f(z)$ is decreasing for real $z, z_{0} \leqq z<1$.

Proof. This follows from 6.3 , since for $z_{0}<z<1$ we have $z f^{\prime \prime}(z) / f^{\prime}(z)$ $+1 \leqq 0$. (See also 5.5.)

Lemma 6.9. $z f^{\prime}(z) / f(z) \leqq 1$ for $0 \leqq z \leqq 1, z$ real.

Proof. $z f^{\prime}(z) / f(z)=1$ for $z=0$ and the rest follows from 6.1 and 6.8.

LEMMA 6.10. $z / f(z)$ is increasing for $0 \leqq z \leqq 1$, real $z$.

Proof. $d[z / f(z)] / d z=(1 / f(z))\left[1-z f^{\prime}(z) / f(z)\right]$ by 6.9 .

ThEOREM 6.11. $f^{\prime}(z)$ is decreasing for real $z, 0 \leqq z \leqq 1$.

Proof. By 6.1 and 6.8, $z f^{\prime}(z) / f(z)$ is decreasing for real $z$, and by $6.10, z / f(z)$ is increasing for this range of $z$. But $z f^{\prime}(z) / f(z)=f^{\prime}(z)$ $\cdot z / f(z)$ which is decreasing for real $z, 0 \leqq z \leqq 1$.

From 6.9 we obtain

$$
z_{0} f^{\prime}\left(z_{0}\right) / f\left(z_{0}\right)=z_{0} f^{\prime}\left(z_{0}\right) / d_{0} \leqq 1
$$

or

$$
z_{0} f^{\prime}\left(z_{0}\right) \leqq d_{0}
$$

7. A lower bound for $d_{0} / d^{*}$ for functions discussed in $\$ 5$. These functions have $d_{0}$ and $d^{*}$ on the positive axis of reals. If $z=z_{0}$ is mapped into $d_{0}$, then we have

$$
\operatorname{Re}\left\{\frac{z_{0} f^{\prime \prime}\left(z_{0}\right)}{f^{\prime}\left(z_{0}\right)}\right\}=-1
$$

and on account of realness

7.2

$$
z_{0} f^{\prime \prime}\left(z_{0}\right)=-f^{\prime}\left(z_{0}\right)
$$

By 5.5 we have for $z>z_{0}$

$$
z_{0} f^{\prime \prime}\left(z_{0}\right) \leqq-f^{\prime}\left(z_{0}\right) .
$$

From this we obtain by integration along the real axis from $z=z_{0}$ :

7.4

$$
f^{\prime}\left(z_{0}\right) \leqq z_{0} f^{\prime}\left(z_{0}\right) / z
$$

and therefore 


$$
d^{*}=\int_{0}^{1} f^{\prime}(z) d z=\int_{0}^{z_{0}} f^{\prime}(z) d z+\int_{z_{0}}^{1} f^{\prime}(z) d z
$$

$$
\begin{aligned}
& =d_{0}+\int_{z_{0}}^{1} f^{\prime}(z) d z \leqq d_{0}+z_{0} f^{\prime}\left(z_{0}\right) \int_{z_{0}}^{1} \frac{d z}{z} \\
& =d_{0}+z_{0} f^{\prime}\left(z_{0}\right) \ln \left(1 / z_{0}\right) .
\end{aligned}
$$

By 6.13 we obtain

$$
d^{*}<d_{0}+d_{0} \ln \left(1 / z_{0}\right)
$$

or

$$
\frac{d_{0}}{d^{*}}>\frac{1}{1+\ln \left(1 / z_{0}\right)}
$$

The right-hand side of 7.7 is a strictly increasing function of $z_{0}$, for $0<z_{0}<1$. Since $r_{0} \geqq 2-3^{1 / 2}$ (see introduction) therefore

$$
\frac{d_{0}}{d^{*}} \geqq \frac{1}{1+\ln \left(1 /\left(2-3^{1 / 2}\right)\right)}=.432 \cdots .
$$

From 7.7 we also notice that if $z_{0} \geqq e^{-1 / 2}=.606 \cdots$, then

$$
d_{0} / d^{*}>2 / 3 \text {. }
$$

8. An application of the Schwarzian derivative. The results obtained so far made use only of elementary methods. If we apply a property of the Schwarzian derivative that was discussed in a recent paper [5], then the results of $\$ 7$ can be improved. We have:

$$
d_{0}=f\left(z_{0}\right)=\int_{0}^{z_{0}} f^{\prime}(z) d z ;
$$

integrating twice by parts, we get:

$$
d_{0}=z_{0} f^{\prime}\left(z_{0}\right)-\frac{1}{2} z_{0}^{2} f^{\prime \prime}\left(z_{0}\right)+\frac{1}{2} \int_{0}^{z_{0}} z^{2} f^{\prime \prime \prime}(z) d z .
$$

But by 7.2 we have $z_{0} f^{\prime \prime}\left(z_{0}\right)=-f^{\prime}\left(z_{0}\right)$ and therefore 8.2 is equivalent to

$$
d_{0}=\frac{3}{2} z_{0} f^{\prime}\left(z_{0}\right)+\frac{1}{2} \int_{0}^{z_{0}} z^{2} f^{\prime \prime \prime}(z) d z .
$$

Now, by (5) we have

$$
|\{f(z), z\}| \equiv\left|\frac{f^{\prime \prime \prime}(z)}{f^{\prime}(z)}-\frac{3}{2}\left[\frac{f^{\prime \prime}(z)}{f^{\prime}(z)}\right]^{2}\right| \leqq 6\left(1-|z|^{2}\right)^{-2}
$$


to be a necessary condition for $f(z)$ to be schlicht. Therefore we have a fortiori

8.5

$$
\left|\operatorname{Re}\left\{\frac{f^{\prime \prime \prime}(z)}{f^{\prime}(z)}-\frac{3}{2}\left[\frac{f^{\prime \prime}(z)}{f(z)}\right]^{2}\right\}\right| \leqq 6\left(1-|z|^{2}\right)^{-2}
$$

and in our case-real coefficients, real, positive $z$-we have

$$
\begin{aligned}
\frac{3}{2}\left[\frac{f^{\prime \prime}(z)}{f^{\prime}(z)}\right]^{2}-6\left(1-z^{2}\right)^{-2} & \\
& \leqq \frac{f^{\prime \prime \prime}(z)}{f^{\prime}(z)} \leqq \frac{3}{2}\left[\frac{f^{\prime \prime}(z)}{f^{\prime}(z)}\right]^{2}+6\left(1-z^{2}\right)^{-2}
\end{aligned}
$$

Since $f^{\prime}(z)$ is positive on the real positive axis, therefore we have from 8.6

$$
f^{\prime \prime \prime}(z) \geqq-6\left(1-z^{2}\right)^{-2} f^{\prime}(z) .
$$

Substituting this value for $f^{\prime \prime \prime}(z)$ into 8.3 we obtain:

8.8

$$
d_{0} \geqq \frac{3}{2} z_{0} f^{\prime}\left(z_{0}\right)-3 \int_{0}^{z_{0}} z^{2}\left(1-z^{2}\right)^{-2} f^{\prime}(z) d z .
$$

Integrating by parts and transposing, we get:

8.9

$$
\left[1+3 z_{0}^{2}\left(1-z_{0}^{2}\right)^{-2}\right] d_{0}
$$

$$
\geqq \frac{3}{2} z_{0} f^{\prime}\left(z_{0}\right)+6 \int_{0}^{z_{0}} z\left(1+z^{2}\right)\left(1-z^{2}\right)^{-3} f(z) d z .
$$

But by 3.5 we have $f(z) \geqq z d^{*}$. Substituting this value for $f(z)$ into the integral of 8.9 and evaluating it we get:

$$
\left[1+3 z_{0}^{2}\left(1-z_{0}^{2}\right)^{-2}\right] d_{0}
$$

$$
\geqq \frac{3}{2} z_{0} f^{\prime}\left(z_{0}\right)+\left[\frac{3}{4} \ln \frac{1+z_{0}}{1-z_{0}}+\frac{3}{2} z_{0} \frac{3 z_{0}^{2}-1}{\left(1-z_{0}^{2}\right)^{2}}\right] d^{*} .
$$

Solving for $z_{0} f^{\prime}\left(z_{0}\right)$ and substituting into 7.5 we get:

$$
d^{*} \leqq d_{0}+\left\{\frac{2}{3} \frac{1+z_{0}^{2}+z_{0}^{4}}{\left(1-z_{0}^{2}\right)^{2}} d_{0}\right.
$$

$$
\left.-\left[\frac{1}{2} \ln \frac{1+z_{0}}{1-z_{0}}+z_{0} \frac{3 z_{0}^{2}-1}{\left(1-z_{0}^{2}\right)^{2}}\right] d^{*}\right\} \ln \left(\frac{1}{z_{0}}\right) \text { or }
$$




$$
\frac{d_{0}}{d^{*}} \geqq \frac{1+\left[\frac{1}{2} \ln \frac{1+z_{0}}{1-z_{0}}+z_{0} \frac{3 z_{0}^{2}-1}{\left(1-z_{0}^{2}\right)^{2}}\right] \ln \left(\frac{1}{z_{0}}\right)}{1+\frac{2}{3} \frac{1+z_{0}^{2}+z_{0}^{4}}{\left(1-z_{0}^{2}\right)^{2}} \ln \left(\frac{1}{z_{0}}\right)}
$$

The function of $z_{0}$ represented by the right-hand side of 8.12 is monotone increasing for $2-3^{1 / 2} \leqq z_{0} \leqq .50 \cdots$. For $z_{0}=2-3^{1 / 2}$ we find

$$
\frac{d_{0}}{d^{*}} \geqq .4962 \ldots
$$

which improves 7.8 .

8.14 A comparison between the results of 7.7 and 8.12. Denoting the right-hand sides of 7.7 and 8.12 by $f_{1}(z)$ and $f_{2}(z)$ respectively, we obtain the following comparison:

\begin{tabular}{l|l|l}
\hline$z$ & $f_{1}(z)$ & $f_{2}(z)$ \\
\hline $2-3^{1 / 2}$ & .4314 & .4962 \\
.30 & .4538 & .5107 \\
.35 & .4878 & .5320 \\
.40 & .5218 & .5518 \\
.45 & .5560 & .5730 \\
.50 & .5906 & .5903 \\
.60 & .6617 & .6316 \\
\hline
\end{tabular}

Acknowledgements. I wish to thank Dr. H. A. Rademacher for having suggested this problem and for very helpful criticism on several points in this paper.

\section{BIBLIOGRAPHY}

1. E. Study, Vorlesungen iber ausgewählte Gegenstdinde der Geometrie, Part 2.

2. Gronwall, C. R. Acad. Sci. Paris, vol. 163 (1916) pp. 249-252.

3. R. Nevanlinna, Oversigt av Finska Vet. Soc. Forh. (1919-20).

4. Pflanz, Math. Zeit. vol. 40 (1935).

5. Z. Nehari, Bull. Amer. Math. Soc. vol. 55 (1949) pp. 545-546.

University of Pennsylvania 